\title{
Placenta percreta with bladder invasion: managed successfully
}

\begin{abstract}
Placenta percreta (PP) is a potentially life-threatening condition. When PP is complicated by bladder invasion, mortality rates have been estimated as high as $9.5 \%$ and $24 \%$ for mother and child, respectively. Early diagnosis allows for appropriate antenatal care and accordingly surgical planning and management. Herein, we are going to present a case report, in which a woman G3P2L2 with previous 2 caesarean section with pre-term gestation was diagnosed with placenta accreta, but intra-operatively it was placenta percreta with bladder invasion. Patient was managed successfully by doing subtotal hysterectomy. Any pregnant patient with previous history of caesarean section we should be suspicious of placenta accrete spectrum, hence should be managed in a tertiary care center with a multidisciplinary approach, and with earliest possible involvement of the urologist in decision-making.
\end{abstract}

Volume 12 Issue 3 - 2021

\author{
Prativa Sadangi, Kumari Ranjeeta \\ Ispat genaral Hospital, Rourkela, India
}

Correspondence: Dr Kumari Ranjeeta, Ispat genaral Hospital, Rourkela, Igh, sector 19, Rourkela, India,

Email ranjita78700@gmail.com

Received: May 03, 202I | Published: May 21, 2021

\section{Introduction}

Placenta accrete is a generalized term used when an abnormal, firmly adherent placenta implants with some degree of invasion into the uterus. It occurs as a result of partial or complete absence of decidua basal is and defective formation of the Nitabuch layer. Placenta percreta, a rare disorder of placental implantation, placental villi penetrate through the decidua and myometrium, even through the serosa of the uterus and into surrounding organs. Placenta villi fully penetrate the myometrium and it invades surrounding structures like bladder, broad ligament or sigmoid colon.

Placenta percreta with bladder invasion may cause massive maternal bleeding, leading to complications of coagulopathy, infection and even death.

Placenta percreta (PP) is a potentially fatal condition, and mortality rate is correlated to the extent of involvement of surrounding structures. When PP is complicated by bladder invasion, mortality rates have been estimated as high as $9.5 \%$ and $24 \%$ for mother and child, respectively. ${ }^{1}$

It is associated with several clinical situations such as previous cesarean delivery, placenta previa, grand multiparity, previous uterine curettage, and previously treated Asherman syndrome, which is a condition characterized by the presence of scars within the uterine cavity. ${ }^{2}$ In clinical practice, Placenta accrete, placenta increta \& placenta percreta ,these variants are encountered in an approximate ratio of $80: 15: 5$ respectively.

Early diagnosis allows for appropriate surgical planning. Herein, we are going to present a case report, followed by a discussion of the alternatives for diagnosis and management of placenta percreta.

\section{Case report}

A 31year female gravida 3, para 2 with 31 week 2 days pregnancy admitted with complaint of bleeding per vaginum since morning. She had previous two cesarean sections and recently diagnosed with hypothyroidism. she was booked case of our institute, diagnosed with placenta previa in 2nd trimester at 18 week.on repeat USG after admission, transabdominal sonographic evaluation for foetal wellbeing revealed a viable fetus with appropriate biometrical parameters and normal amniotic fluid but suspicious of placenta accreta. The patient was managed conservatively till 34 weeks by giving antibiotics and steroids coverage prophylactically, decision for elective caesarean section \& consent for caesarean hysterectomy was taken as per need. A multidisciplinary team of most experienced obstetricians, anesthetists and neonatologists was present during surgery. A longitudinal midline infraumbilical Incision given over lower abdomen, after opening the abdomen lower segment area was showing typical feature of? percreta and uterus opened by giving transverse incision over upper segment to bypass placenta as placenta was anterior and covering os completely. A healthy, pre-term $2220 \mathrm{~g}$ male baby was delivered out by vertex with Apgar scores of 10 and 10. Placenta removal not attempted and left it in situ. We immediately proceeded with an emergency hysterectomy, during which the lower uterine segment was found to be densely adherent to the bladder wall. During uterine artery clamping, at the time of cutting vesico peritoneal fold \& pushing the bladder downward, placental tissue started bleeding. In the meantime post. Wall of bladder got opened up \& there was e/o torrential bleeding.

Surgeon call was given for bladder repair and repair was done. At the same it was seen that placental tissue invading bladder wall \&tissue between bladder and cervix was relatively indistinguishable. Cervix could not be approached \&subtotal hysterectomy was done. The bladder was repaired in two layers with Vicryl 2-0 suture in doing continuous suturing and bladder was irrigated to ensure water-tight closure. Procedure was subsequently terminated after placement of Foley's catheter and an intraperitoneal drain. Intraoperatively 11 units of various blood and blood products were transfused in total. Shifted to the intensive care unit immediately post-op, transferred to the ward on day 3 , got 2 unit blood product post-operatively on day 3 and discharged uneventfully on postoperative day 18 .

The Foley catheter was left in place for 6 week and the patient has not experienced further urinary difficulties on follow-up.

\section{Discussion}

PAS is a rare complication of pregnancy affecting 1.7 per 10,000 pregnant women, but now a days incidence is rising as a consequence of rising CS rate. ${ }^{3}$ It is seen that antenatal diagnosis of PAS leads to 1.45 fewer units of blood transfusion. ${ }^{4}$ 
Risk of placenta percreta increases with no. of previous CS. ${ }^{5}$ The most recent data from UK indicate that even in presence of clearly recognizable risk factors, only about $50 \%$ of PAS diagnosed prior to delivery but rate of hysterectomy is $>50 \%$.

The incidence of concomitant bladder invasion is much lower, occurring in approximately 1 in 10,000 births. ${ }^{6}$

Current prenatal diagnosis based upon subjective interpretation of typical sonographic finding \&colour Doppler imaging,magnetic resonance imaging (MRI), and cystoscopy. MRI may reveal nonvisualization of the inner layer of the placenta-myometrium interface on half-Fourier single-shot turbo spin-echo images. ${ }^{7}$ Cystoscopy may often show posterior bladder wall abnormalities can be seen by. Fulguration and/or biopsy of these abnormalities should be avoided, as this may precipitate massive hemorrhage. ${ }^{8}$ USG must be carried out with a full bladder $(200-300 \mathrm{ml})$.

Management includes diagnosis should be made before 30 weeks gestation to plan elective delivery at $35^{+0}$ to $36^{+6}$ weeks gestation and managed must be at tertiary care center.

The risk of PP includes massive haemorrhage, blood transfusion, risk of hysterectomy, lower urinary tract damage, infection and maternal death even.

The management of placenta percreta can be challenging as the average blood loss during delivery is 3 liter or more in $90 \%$ patients. ${ }^{9}$ Forty percent of the patients need $>10$ transfusions it has a $7 \%$ maternal mortality rate, ${ }^{6}$ as seen in our case, i.e pt. needed 11 transfusions intraoperatively. Refractory hematuria is present in $25 \%$ patients due to bladder invasion by placenta percreta. ${ }^{8}$

Delivery of baby by CS in PP should be done by opening the uterus at a site distant from the placenta $\&$ delivering baby without disturbing the placenta.

CS hysterectomy with placenta left in-situ is preferable to attempting to separate it from uterus. If a diagnosis of placenta percreta is made after delivery of the baby, a CS hysterectomy with placenta left in-situ is preferable to attempting to separate it from uterus. Chandraharan et al introduced a Triple-P procedure as a conservative surgical alternative to peripartum hysterectomy for placenta percreta. It includes perioperative placental localization, pelvic devascularisation \& placental non-separation with myometrial excision. Neither methotrexate nor arterial embolization reduces the risk of bleeding and infection \& hence neither is recommended routinely.

Follow up is done by ultrasound and serum beta-human chorionic gonadotropin measurements.

\section{Conclusion}

Any pregnant patient with previous history of caesarean section we should be suspicious of placenta accrete spectrum. Placenta percreta with bladder invasion is a life-threatening condition for both mother and baby, hence should be managed in a tertiary care center with a multidisciplinary approach, and with early involvement of the urologist in decision-making. Each option for the management of such cases should be weighed, discussed with the patient and family, and planned in detail preoperatively. Mismanagement of such difficult situations can have potentially devastating consequences. There is need of some more exploration in this field so that, conservative measures should be adopted.

\section{Acknowledgments}

None.

\section{Funding}

None.

\section{Conflicts of interest}

The authors declare no conflicts of interest in preparing this article.

\section{References}

1. Price FV, Resnik E, Heller KA, et al. Placenta previa percreta involving the urinary bladder: a report of two cases and review of the literature. Obstet Gynecol. 1991;78:508-511.

2. Poggi SBH, Kapernick PS. Postpartum hemorrhage and abnormal puerperium. In: DeCherney AH, Nathan L, editors. Current Diagnosis and Treatment Obstetrics and Gynecology. 10th edn. New York: McGraw-Hill Medical; 2007.

3. Fitzpatrick KE, Sellers $S$, Spark $P$ et al Incidence and risk factors for placenta accreta/increta/percreta in the UK: a national case-control study. PLoS one. 2012;7e52893.

4. Buca D, Liberati M, Calì G, et al. Influence of prenatal diagnosis of abnormally invasive placenta on maternal outcome.systemic review \& meta-analysis. Ultrasound Obstet Gynecol. 2018;52;289-416.

5. Silver RM, Landon MB, Rouse DJ, et al. Maternal morbidity associated associated with multiple repeat cesarean deliveries. Obstet Gynecol. 2006;107:1226-1232.

6. Abbas F, Talati J, Wasti S. Placenta percreta with bladder invasion as a cause for life threatening hemorrhage. J Urol. 2000;164:1270-1274.

7. Comstock $\mathrm{CH}$. Antenatal diagnosis of placenta accreta: a review. Ultrasound Obstet Gynecol. 2005;26:89-96.

8. Takai N, Eto M, Sato F, et al. Placenta percreta invading the urinary bladder. Arch Gynecol Obstet. 2005;271:274-275.

9. Hudon L, Belfort MA, Broome DR. Diagnosis and management of placenta percreta: a review. Obstet Gynecol Surv. 1998;53:509-517. 